\title{
Switching From Pregabalin to Mirogabalin in Patients with Peripheral Neuropathic Pain: A Multi-Center, Prospective, Single-Arm, Open-Label Study (MIROP Study)
}

Yoshiyuki Kimura - Shigeki Yamaguchi - Takahiro Suzuki - Jitsu Kato · Satoko Chiba •

Naomi Hirakawa $\cdot$ Keisuke Yamaguchi $\cdot$ Yutaka Tanabe $\cdot$ Hiroshi Takatsuna $\cdot$ Yusuke Kenyoshi

Kazuhito Shiosakai $\cdot$ Miyoshi Sakai $\cdot$ Masako Iseki

Received: December 24, 2020 / Accepted: March 10, 2021 / Published online: April 15, 2021

(c) The Author(s) 2021

\section{ABSTRACT}

Introduction: Mirogabalin, which is a selective ligand of the $\alpha_{2} \delta$ subunit of voltage-gated $\mathrm{Ca}^{2+}$ channels, was recently approved in Japan for peripheral neuropathic pain. The $\alpha_{2} \delta$ ligands, including mirogabalin and pregabalin, are

Supplementary Information The online version contains supplementary material available at https:// doi.org/10.1007/s40122-021-00255-y.

Y. Kimura $(\bowtie) \cdot$ S. Yamaguchi

Department of Anesthesiology, Dokkyo Medical University School of Medicine, 880 Kitakobayashi, Mibu-machi, Shimotsuga-gun, Tochigi 321-0293, Japan

e-mail: y-kimura@dokkyomed.ac.jp

T. Suzuki $\cdot$ J. Kato

Department of Anesthesiology, Nihon University School of Medicine, 30-1 Oyaguchi Kami-cho, Itabashi-ku, Tokyo 173-8610, Japan

S. Chiba $\cdot$ M. Iseki

Department of Anesthesiology and Pain Medicine, Juntendo University Faculty of Medicine, 3-1-3

Hongo, Bunkyo-ku, Tokyo 113-8431, Japan

N. Hirakawa

Pain Clinic and Palliative Care Medicine, Saga University Hospital, 5-1-1 Nabeshima, Saga City, Saga 849-8501, Japan

K. Yamaguchi

Department of Anesthesiology and Pain Medicine, Juntendo Tokyo Koto Geriatric Medical Center, 3-320 Shinsuna, Koto-ku, Tokyo 136-0075, Japan associated with significant risk of adverse events (AEs) such as somnolence or dizziness, leading to poor compliance and subsequent inefficacy. Safety and efficacy data for switching patients from pregabalin to mirogabalin are scarce.

Methods: This prospective, single-arm, openlabel study involving ten participating centers in Japan recruited patients aged $\geq 20$ years with peripheral neuropathic pain [visual analog scale (VAS) score $\geq 40 \mathrm{~mm}$. Where necessary, patients underwent a 1-week tapering period to reduce their pregabalin dose, after which

Y. Tanabe

Anesthesiology and Pain Medicine, Juntendo

University Nerima Hospital, 3-1-10 Takanodai,

Nerima-ku, Tokyo 177-8521, Japan

H. Takatsuna $\cdot$ Y. Kenyoshi

Medical Science Department, Medical Affairs

Division, Daiichi Sankyo Co., Ltd., 3-5-1,

Nihonbashi Honcho, Chuo-ku, Tokyo 103-8426, Japan

K. Shiosakai

Data Intelligence Department, Digital

Transformation Management Division, Daiichi

Sankyo Co., Ltd., 1-2-58, Hiromachi, Shinagawa-ku, Tokyo 140-8710, Japan

M. Sakai

EP-CRSU Co., Ltd., Acropolis Tokyo 3F 6-29 ShinOgawamachi Shinjuku-ku, Tokyo 162-0814, Japan 
pregabalin was stopped and mirogabalin dose was increased using a step-wise dose titration. Patients underwent dose increases after the first and second weeks if there were no tolerability issues, followed by the effective doses until the end of the study (4 weeks). The primary endpoint was the incidence of somnolence, dizziness, and peripheral edema; secondary endpoints included changes in VAS score. AEs were monitored for safety.

Results: Of 157 patients who provided informed consent, 152 patients were enrolled; $136(89.5 \%)$ patients completed the study. The overall incidences of somnolence, dizziness, and peripheral edema were 41.4, 15.8, and $2.6 \%$, respectively. Most patients (>70\%) experienced mild AEs, and one patient experienced a severe $\mathrm{AE}$ (dizziness). Most patients $(>70 \%)$ were able to achieve dose titration to an effective dose. Overall mean VAS score significantly decreased $(\Delta 15.7 \mathrm{~mm}, p<0.0001)$ by the end of the study.

Conclusions: Mirogabalin switching from pregabalin is well tolerated and effective in pain management for peripheral neuropathic pain using a step-wise titration.

Trial Registration: Japan Registry of Clinical Trials (jRCTs031190113).

Keywords: Mirogabalin; Peripheral neuropathic pain; Pregabalin; Switching; $\alpha_{2} \delta$ ligands

\section{Key Summary Points}

\section{Why carry out this study?}

Mirogabalin, a potent selective ligand of the $\alpha_{2} \delta$ subunit of voltage-gated $\mathrm{Ca}^{2+}$ channels, has been approved in Japan for the treatment of peripheral neuropathic pain.

Patients with peripheral neuropathic pain treated with $\alpha_{2} \delta$ ligand such as pregabalin and mirogabalin may experience side effects including somnolence and dizziness, leading to poor compliance and subsequent inefficacy.
We conducted this single-arm, open-label study to assess the safety and efficacy of switching from pregabalin to mirogabalin in patients with peripheral neuropathic pain under conditions similar to clinical practice.

\section{What was learned from the study?}

Switching from pregabalin at or below the initial dose to mirogabalin was generally well tolerated and the pain intensity was decreased.

Most (> 70\%) patients were able to increase to an effective dose of mirogabalin by a step-wise dose titration.

Switching from pregabalin to mirogabalin may be an available option although patients should be monitored carefully for somnolence and dizziness.

\section{DIGITAL FEATURES}

This article is published with digital features, including a summary slide, to facilitate understanding of the article. To view digital features for this article go to https://doi.org/10.6084/ m9.figshare.14179217.

\section{INTRODUCTION}

According to the International Association for the Study of Pain, neuropathic pain is defined as "pain caused by a lesion or disease of the somatosensory nervous system" [1]. Neuropathic pain is caused by various pathological conditions such as diabetes, viral infection, and spinal canal stenosis and leads to multiple symptoms, including spontaneous neurological pain, allodynia, and hyperalgesia. The severity and duration of symptoms vary depending on the etiology [2,3], and treatment is challenging [4]. Patients with neuropathic pain show higher ratings for pain intensity with more comorbidities, such as depression, panic/anxiety 
disorder, and sleep disorders than those with nociceptive pain, which can negatively impact their quality of life [5].

Mirogabalin, a selective oral $\alpha_{2} \delta$ ligand, was first approved for peripheral neuropathic pain in 2019 in Japan [6]. Mirogabalin was shown to be effective and well tolerated in the management of postherpetic neuralgia [7] and diabetic peripheral neuropathic pain in Asian patients [8].

In vitro studies of its pharmacologic action have shown that mirogabalin has a higher binding affinity than pregabalin for the human and rat $\alpha_{2} \delta$ subunits, an element of voltagegated $\mathrm{Ca}^{2+}$ channels and the target through which these drugs elicit their analgesic effect [9]. In vivo models, such as rats with partial sciatic nerve ligation and streptozotocin-induced diabetes, support this mechanism, as the analgesic effects of mirogabalin were stronger and lasted longer in these rats [9]. In a dissociation rate analysis, the dissociation half-lives of mirogabalin from the $\alpha_{2} \delta-1$ and $\alpha_{2} \delta-2$ subunits were $11.1 \mathrm{~h}$ and $2.4 \mathrm{~h}$, respectively, compared with $1.4 \mathrm{~h}$ for pregabalin at both subunits [9].

Both mirogabalin and pregabalin carry significant risk of adverse events (AEs) such as somnolence, dizziness, peripheral edema, and weight gain, which are listed as the most common treatment-emergent AEs [7, 8, 10, 11]. These AEs can significantly lower compliance, sometimes leading to discontinuation before a therapeutic effect can be achieved. In patients in whom insufficient efficacy is observed with pregabalin, switching to other agents has been studied, and a reduction in AEs and an improvement in pain relief have been reported [12]. Thus, when considering a switch from pregabalin to mirogabalin, safety is an important consideration; however, there are insufficient safety data available in this setting.

In previous clinical trials investigating mirogabalin, patients who were previously taking pregabalin underwent a washout period; however, the safety of directly switching from pregabalin to mirogabalin was not examined $[7,8]$. Furthermore, in a retrospective analysis that examined the switch from pregabalin to mirogabalin, information on the washout period was not reported [13].
For patients and prescribing physicians, it is important to understand the safety of switching from pregabalin to mirogabalin under conditions close to actual clinical practice. Therefore, this clinical study was designed as an exploratory evaluation of the safety (focusing on somnolence, dizziness, and peripheral edema) and efficacy of mirogabalin when switching from pregabalin to mirogabalin in patients with peripheral neuropathic pain whose pain was moderate or greater (according to the visual analog scale [VAS]), and who had been receiving pregabalin for at least 4 weeks.

\section{METHODS}

\section{Trial Design}

This was a multi-center, prospective, single-arm, open-label study, which took place in 10 centers in Japan between 1 October 2019 and 30 April 2020 (details are listed in the electronic supplementary material). The study received approval from the Clinical Research Review Committee, Itabashi Hospital, Nihon University School of Medicine (CRB No. CRB3180013), which notified all participating centers of the approval. The trial was conducted in accordance with the ethical principles, clinical research laws, and relevant notifications stipulated in the Declaration of Helsinki (as revised in 2013). This study was registered in the Japan Registry of Clinical Trials under the identifier jRCTs031190113.

After obtaining informed consent, where necessary the dose of pregabalin was gradually reduced over 1 week according to the package insert [11], to reduce the risk of withdrawal symptoms. Patients with creatinine clearance $(\mathrm{CrCL}) \geq 60 \mathrm{ml} / \mathrm{min}$ reduced their daily pregabalin dose to $\leq 150 \mathrm{mg}$, and patients with CrCL 30 to $<60 \mathrm{ml} / \mathrm{min}$ to $\leq 75 \mathrm{mg}$; the schedule of dose reduction was at the discretion of the attending physician. At enrollment, pregabalin was stopped completely and administration of mirogabalin was initiated. Mirogabalin was titrated according to renal function (Fig. 1). For patients with $\mathrm{CrCL} \geq 60 \mathrm{ml} / \mathrm{min}$, mirogabalin was administered as follows: $5 \mathrm{mg}$ twice daily 


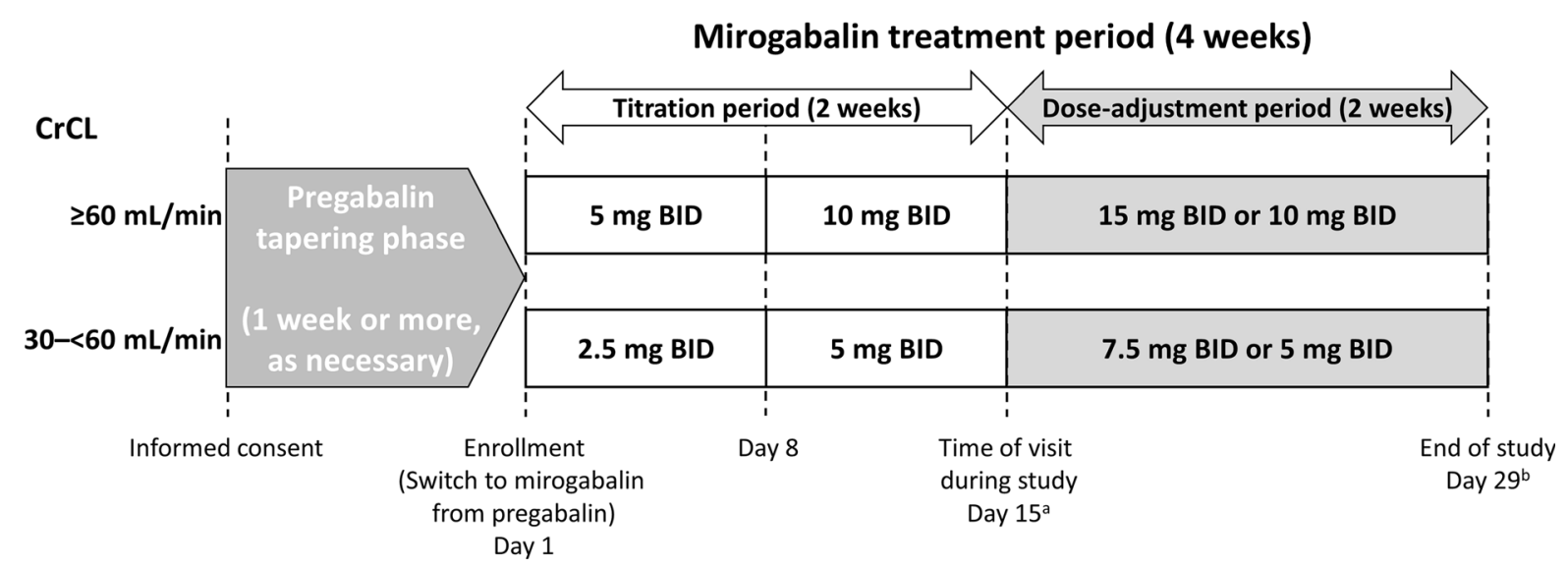

Fig. 1 Study design. ${ }^{a} \pm 7$ days; i.e., dose-adjustment period could begin anywhere between days 9 and $22 .{ }^{b} \pm 7$ days; i.e., end of study occurred anywhere between days 22 and 36. BID twice daily, $\operatorname{Cr} C L$ creatinine clearance

(BID) for 1 week, $10 \mathrm{mg}$ BID for 1 week, and $15 \mathrm{mg}$ BID or $10 \mathrm{mg}$ BID for 2 weeks. For patients with $\mathrm{CrCL} 30$ to $<60 \mathrm{ml} / \mathrm{min}$, mirogabalin was administered as $2.5 \mathrm{mg}$ BID (1 week), $5 \mathrm{mg}$ BID (1 week), and $7.5 \mathrm{mg}$ BID or $5 \mathrm{mg}$ BID ( 2 weeks). From the time of informed consent to the end of the study (or discontinuation), changes to, or additions of, new pain drugs/therapies were avoided as much as possible.

\section{Patients}

The investigators explained the participation in this clinical study to patients who were considered suitable subjects. When the patient consented to participate, the investigator reduced the amount of pregabalin as required, and then performed a screening test. Demographics, medical history, confirmation of drugs used for 28 days prior to enrollment, vital signs, CrCL, and VAS pain intensity were recorded on a standard $100-\mathrm{mm}$ scale by the sub-investigator during screening. Throughout the study, pain intensity, symptoms, AEs, and use of any other drugs were recorded by the physician at each visit, and at the time of study discontinuation. The eligibility criteria were confirmed based on the results of the screening test, and patients who met the eligibility criteria were enrolled as study participants.
The inclusion and exclusion criteria were as follows. Patients with a diagnosis of peripheral neuropathic pain according to the judgment of a physician with specialized training in pain management, who had been taking pregabalin for $\geq 28$ days, had a VAS score [14] of $\geq 40 \mathrm{~mm}$ at the time of informed consent and at enrollment, and were aged $\geq 20$ years at the time of informed consent were eligible for this study. Although the diagnostic method for peripheral neuropathic pain was not specified in this study, in Japanese clinical practice, the guidelines published by the Japan Society of Pain Clinicians are typically used [15]. Specifically, the guidelines contain a neuropathic pain diagnostic algorithm, which is the grading system created by the International Association for the Study of Pain [16]. Patients who agreed to participate in the study were required to be able to understand the procedures of the study and answer questions appropriately. Patients were excluded if they had a history of hypersensitivity to the components of pregabalin or mirogabalin; had concomitant serious liver, kidney, or heart disease; had $\mathrm{CrCL}<30 \mathrm{ml} / \mathrm{min}$ by the Cockcroft-Gault equation; were pregnant or breastfeeding; or were deemed by the treating physician or study investigator as unsuitable for study participation. The use of new concomitant drugs or therapies for the management of pain was not allowed from the time of obtaining informed consent to the end 
of the study. All patients provided written informed consent.

\section{Endpoints}

The primary endpoints were the incidences of somnolence, dizziness, and peripheral edema from enrollment to the end of the study (safety analysis set). These AEs were recorded through an active listening process during physicianconducted interviews with the patients. Patients were first asked whether they had any concerns about side effects in general, and were then asked specifically whether they had experienced somnolence, dizziness, or peripheral edema. Patients were then asked whether there were any other side effects that they were worried about. The secondary endpoints were changes in pain intensity (VAS score) from enrollment (day 1) to the end of the study (day 29) and at the second visit (mid-study, day 15). VAS scores were obtained from patients' subjective reports of pain intensity, with "no pain" given a score of zero and "worst pain imaginable" given a score of 100. AEs, including the date of onset/ outcome, seriousness, severity, outcome, causality to the study drug, causality to the clinical study, and treatment were recorded by the physician or sub-investigator in case report forms at each visit. AEs were coded according to the Japanese Medical Dictionary for Regulatory Activities (MedDRA/J), version 23.0.

Additional endpoints included the cumulative incidences of somnolence, dizziness, and peripheral edema assessed at days 1, 8, 15, and 29 . The confirmation of symptoms (somnolence, dizziness, and peripheral edema), dosing status of the study drug, and confirmation of concomitant drugs/therapies were assessed at day 1, day 15 (visit window, day 9 to day 22), day 29 (visit window, day 23 to day 36), and at the time of discontinuation.

\section{Statistical Analysis}

The 4-week incidences of somnolence, dizziness, and peripheral edema in each active treatment group during the double-blind phase of a phase 3 study of mirogabalin in postherpetic neuralgia patients were 10.6-22.6, 4.0-12.3, and 0.7-3.9\%, respectively [7]. Assuming a $10 \%$ incidence of AEs in this clinical study for mirogabalin or pregabalin, a sample size of 140 patients was estimated to be necessary to achieve a $95 \%$ confidence interval of \pm $5 \%$ accuracy. Considering dropouts, the target number of patients was set at 150 .

The safety analysis set included all patients who had received at least one dose of mirogabalin. The efficacy analysis set included all enrolled patients who received at least one dose of mirogabalin, were compliant with the study protocol, and for whom there were VAS measurements available at both study enrollment and post-dose.

For categorical variables, numbers and proportions are presented, and for continuous variables, summary statistics [number of patients, mean value, standard deviation (SD), minimum value, quartile, and maximum value] were calculated.

The incidences of somnolence, dizziness, and peripheral edema from the time of enrollment to the end of the study and the 95\% confidence intervals were calculated by the Clopper-Pearson method (safety analysis set). For patients with data not available at day 29 (visit 3), data from their last visit (including the time of discontinuation) were assessed at the end of the study.

For the efficacy analysis set, summary statistics were calculated for the VAS score at informed consent and at days 1, 15, and 29. For change in VAS score from time of enrollment, summary statistics and 95\% confidence intervals were calculated, and $p$ values were calculated using a paired $t$ test.

In the safety analysis set, the cumulative incidences of somnolence, dizziness, and peripheral edema at days $1,8,15$, and 29 were estimated, together with 95\% confidence intervals, using the Kaplan-Meier method. AEs were reported using descriptive statistics.

The primary and secondary endpoints were analyzed according to the following subgroups: VAS at time of enrollment ( $\geq 40$ to $<60 \mathrm{~mm} /$ $\geq 60 \mathrm{~mm}$ ); pregabalin tapering (Yes/No); daily pregabalin dose (tertiles) at the time of informed consent $(<25$ th percentile/25th to $<$ 
50th percentile/50th to $<75$ th percentile/ $\geq 75$ th percentile); concomitant medications (Yes/No); primary target disease (diabetic peripheral neuropathic pain/postherpetic neuralgia/lumbar spinal stenosis/lumbar disc herniation/other peripheral neuropathy/noxious pain); and completion of protocol treatment according to renal function (CrCL) [complete $(\geq 60 \mathrm{ml} / \mathrm{min}) /$ incomplete $(\geq 60 \mathrm{ml} / \mathrm{min}) /$ complete $(30-<60 \mathrm{ml} / \mathrm{min}) /$ incomplete $(30-<$ $60 \mathrm{ml} / \mathrm{min}$ )]. The significance level for hypothesis testing was set at 5\%, and the confidence intervals for both sides was at $95 \%$. The statistical analysis was performed using SAS Version 9.4 (SAS Institute Inc., Cary, NC, USA).

\section{RESULTS}

\section{Patients}

Informed consent was obtained from 157 patients; of these, 152 patients met the eligibility criteria, were enrolled in the study, and were included in the safety analysis set (Fig. 2). The efficacy analysis set consisted of 151 patients. One patient was excluded because no VAS measurement was available after administration of mirogabalin. A total of 136 patients completed the study and 16 patients discontinued the study. The most common reason for discontinuation was AEs (10 patients).

Patient demographics and clinical characteristics for the safety analysis set are shown in Table 1. A similar proportion of male $(52.0 \%)$ and female $(48.0 \%)$ patients were enrolled, with a mean \pm SD age of $66.1 \pm 12.9$ years and a mean \pm SD VAS score of $67.4 \pm 16.9 \mathrm{~mm}$ at enrollment. Most patients $(62.5 \%)$ had a VAS score $>60 \mathrm{~mm}$. Mean $\pm \mathrm{SD}$ CrCL at enrollment was $82.5 \pm 37.4 \mathrm{ml} / \mathrm{min}$, and most patients $(75.0 \%)$ had a CrCL of $\geq 60 \mathrm{ml} / \mathrm{min}$. Pregabalin tapering was performed in 38 patients $(25.0 \%)$. Mean \pm SD pregabalin daily dose in patients overall was $122.7 \pm 75.1 \mathrm{mg} /$ day at informed consent, and $102.0 \pm 44.7 \mathrm{mg} /$ day at enrollment.

For the safety analysis set, concomitant nondrug therapy was received by 70 patients (46.1\%), of whom $59(38.8 \%)$ were receiving nerve block therapy at the time of enrollment. Overall, 133 patients $(87.5 \%)$ were receiving concomitant drug therapy, of whom 69 (45.4\%) received tramadol formulations and 21 (13.8\%) received duloxetine. Postherpetic neuralgia and lumbar spinal stenosis accounted for more than half of the patients with peripheral neuropathic pain (55.3\%); "other peripheral neuropathic pain" accounted for approximately a third of

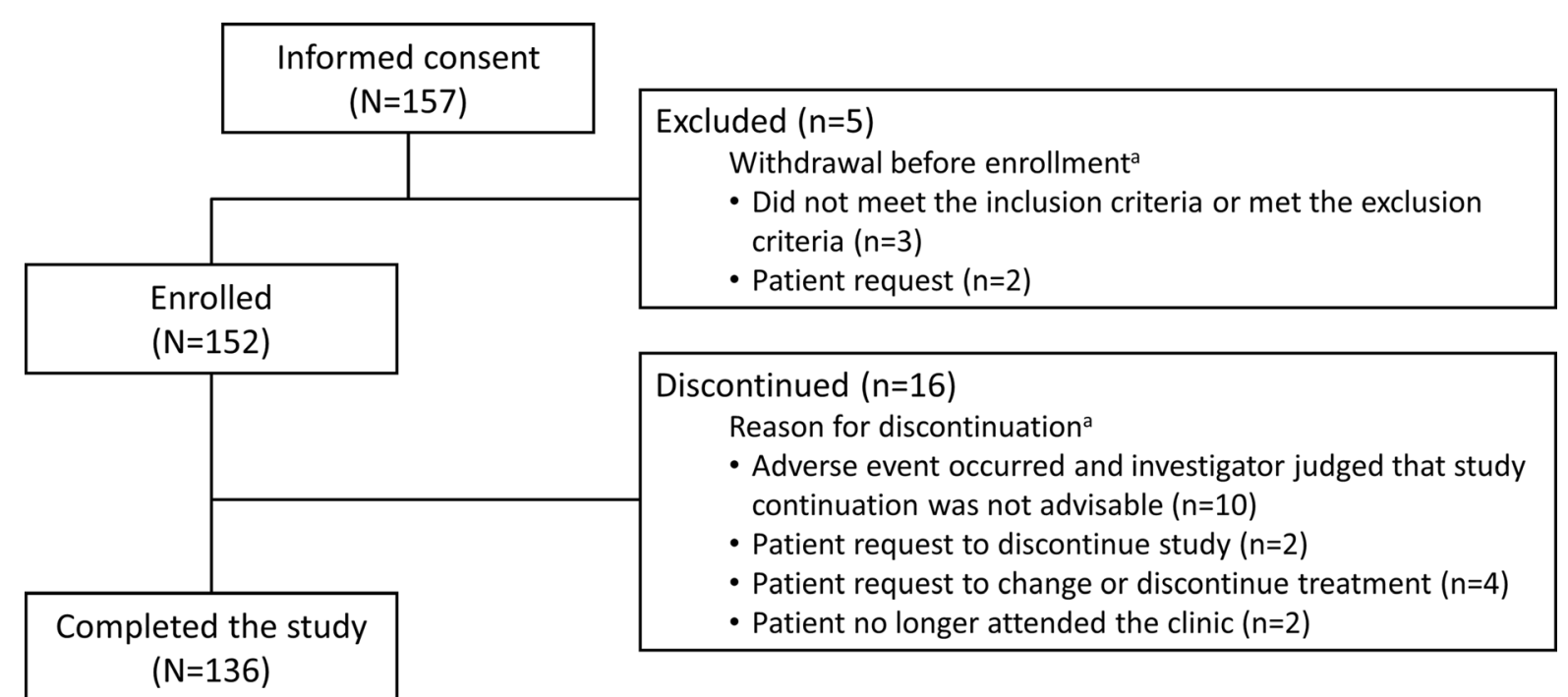

Fig. 2 Patient disposition. ${ }^{\text {a }}$ Patients may have had multiple reasons for withdrawal 
Table 1 Baseline patient demographics and clinical characteristics (safety analysis set)

\begin{tabular}{|c|c|}
\hline Characteristics & $\begin{array}{l}\text { Patients } \\
(N=152)\end{array}$ \\
\hline Age, years & $66.1 \pm 12.9$ \\
\hline \multicolumn{2}{|l|}{ Sex } \\
\hline Male & $79(52.0)$ \\
\hline Female & $73(48.0)$ \\
\hline Body weight, kg & $62.5 \pm 13.0$ \\
\hline $\begin{array}{l}\text { Pain intensity (VAS score) at informed } \\
\text { consent, } \mathrm{mm}\end{array}$ & $66.7 \pm 16.7^{\mathrm{a}}$ \\
\hline VAS score at enrollment, $\mathrm{mm}$ & $67.4 \pm 16.9^{\mathrm{a}}$ \\
\hline $40-<60$ & $57(37.5)$ \\
\hline$\geq 60$ & $95(62.5)$ \\
\hline $\mathrm{CrCL}$ at enrollment ${ }^{\mathrm{b}}, \mathrm{ml} / \mathrm{min}$ & $82.5 \pm 37.4$ \\
\hline$\geq 60$ & $114(75.0)$ \\
\hline $30-<60$ & $38(25.0)$ \\
\hline $\begin{array}{l}\text { Pregabalin daily dose at informed } \\
\text { consent, } \mathrm{mg} / \text { day }\end{array}$ & $122.7 \pm 75.1$ \\
\hline $\mathrm{CrCL} \geq 60 \mathrm{ml} / \mathrm{min}$ & $134.2 \pm 77.9$ \\
\hline $\mathrm{CrCL} 30-<60 \mathrm{ml} / \mathrm{min}$ & $88.2 \pm 53.5$ \\
\hline $\begin{array}{l}\text { Pregabalin daily dose at enrollment, } \\
\mathrm{mg} / \text { day }\end{array}$ & $102.0 \pm 44.7$ \\
\hline $\mathrm{CrCL} \geq 60 \mathrm{ml} / \mathrm{min}$ & $114.7 \pm 44.0$ \\
\hline $\mathrm{CrCL} 30-<60 \mathrm{ml} / \mathrm{min}$ & $63.8 \pm 16.1$ \\
\hline \multicolumn{2}{|l|}{$\begin{array}{l}\text { Pregabalin tapering performed between } \\
\text { informed consent and enrollment }\end{array}$} \\
\hline Yes & $38(25.0)$ \\
\hline No & $114(75.0)$ \\
\hline \multicolumn{2}{|l|}{ Primary target disease } \\
\hline Postherpetic neuralgia & $43(28.3)$ \\
\hline Lumbar spinal stenosis & $41(27.0)$ \\
\hline Lumbar disc herniation & $11(7.2)$ \\
\hline Diabetic peripheral neuropathic pain & $1(0.7)$ \\
\hline Other peripheral neuropathic pain & $56(36.8)$ \\
\hline
\end{tabular}

Table 1 continued

\begin{tabular}{|c|c|}
\hline Characteristics & $\begin{array}{l}\text { Patients }(N \\
=152)\end{array}$ \\
\hline $\begin{array}{l}\text { Diseases other than peripheral } \\
\text { neuropathic pain }\end{array}$ & $0(0.0)$ \\
\hline Unknown & $0(0.0)$ \\
\hline Medical history & $79(52.0)$ \\
\hline Complications & $126(82.9)$ \\
\hline Operation history & $17(11.2)$ \\
\hline Lumbar laminectomy & $5(3.3)$ \\
\hline Lumbar interbody fusion & $4(2.6)$ \\
\hline Lumbar lateral interbody fusion & $0(0.0)$ \\
\hline Other & $12(7.9)$ \\
\hline $\begin{array}{l}\text { Concomitant drug used between } \\
\text { informed consent and enrollment }\end{array}$ & $133(87.5)$ \\
\hline Tramadol formulation & $69(45.4)$ \\
\hline Acetaminophen & $26(17.1)$ \\
\hline Ketoprofen & $24(15.8)$ \\
\hline Duloxetine & $21(13.8)$ \\
\hline Loxoprofen & $19(12.5)$ \\
\hline Mecobalamin & $16(10.5)$ \\
\hline Shakuyaku-kanzo-to ${ }^{\mathrm{d}}$ & $16(10.5)$ \\
\hline $\begin{array}{l}\text { Combination non-drug therapy used } \\
\text { between informed consent and } \\
\text { enrollment }^{\mathrm{e}}\end{array}$ & $70(46.1)$ \\
\hline Nerve block & $59(38.8)$ \\
\hline Exercise & $9(5.9)$ \\
\hline Low-level laser & $7(4.6)$ \\
\hline Phototherapy & $4(2.6)$ \\
\hline Joint injection & $3(2.0)$ \\
\hline Somnolence at enrollment & $24(15.8) / 1$ \\
\hline Mild/Moderate/Severe & $(0.7) / 0(0.0)$ \\
\hline Dizziness at enrollment & $9(5.9) / 0(0.0) / 0$ \\
\hline Mild/Moderate/Severe & $(0.0)$ \\
\hline
\end{tabular}


Table 1 continued

\begin{tabular}{lc}
\hline Characteristics & $\begin{array}{l}\text { Patients }(\boldsymbol{N} \\
=\mathbf{1 5 2})\end{array}$ \\
\hline Peripheral edema at enrollment & $10(6.6) / 0(0.0) /$ \\
Mild/Moderate/Severe & $0(0.0)$
\end{tabular}

Values are shown as $n(\%)$ or mean \pm standard deviation $C r C L$ creatinine clearance; $V A S$ visual analog scale

a $N=151$ for the efficacy analysis set

b $\mathrm{CrCL}$ value at enrollment or within the past 6 months from enrollment

c Used by $>10 \%$ of patients

$\mathrm{d}$ Traditional Japanese herbal medicine

e Used by $>2 \%$ of patients

Table 2 Maximum daily dose of mirogabalin by renal function at enrollment (safety analysis set)

\begin{tabular}{|c|c|c|}
\hline $\begin{array}{l}\text { Mirogabalin } \\
\text { dose }\end{array}$ & $\begin{array}{l}\mathrm{CrCL} \geq 60 \mathrm{ml} / \\
\min \\
(N=114)\end{array}$ & $\begin{array}{l}\text { CrCL } 30 \text { to } \\
<60 \mathrm{ml} / \mathrm{min} \\
(N=38)\end{array}$ \\
\hline $2.5 \mathrm{mg} \mathrm{BID}$ & $0(0.0)$ & $7(18.4)$ \\
\hline $5 \mathrm{mg}$ BID & $16(14.0)$ & $9(23.7)$ \\
\hline $7.5 \mathrm{mg}$ BID & $6(5.3)$ & $22(57.9)$ \\
\hline $10 \mathrm{mg}$ BID & $26(22.8)$ & $0(0.0)$ \\
\hline $15 \mathrm{mg}$ BID & $64(56.1)$ & $0(0.0)$ \\
\hline Other & $2(1.8)$ & $0(0.0)$ \\
\hline
\end{tabular}

Values are shown as $n$ (\%)

$B I D$ twice daily, $C r C L$ creatinine clearance

patients $(36.8 \%)$. Details of other peripheral neuropathic pain are included in Table $S 1$ in the electronic supplementary material. At enrollment, somnolence, dizziness, and peripheral edema were all mild with the exception of one case of somnolence (moderate), and these incidences were $24(15.8 \%)$, nine $(5.9 \%)$, and ten $(6.6 \%)$ patients, respectively. For the safety analysis set, the maximum daily dose of mirogabalin according to renal function is shown in Table 2. Patients who received $10 \mathrm{mg}$
BID and $15 \mathrm{mg}$ BID (effective doses) as a maximum daily dose were $22.8 \%$ and $56.1 \%$ of the patients with normal renal function and mild renal impairment, respectively. In patients with moderate renal impairment, patients who received effective doses were $23.7 \%$ for $5 \mathrm{mg}$ BID and $57.9 \%$ for $7.5 \mathrm{mg}$ BID.

\section{Safety}

The incidences of somnolence, dizziness, and peripheral edema during the study period are shown in Table 3 , with overall incidences of $41.4,15.8$, and $2.6 \%$, respectively. Most cases were mild [somnolence, 48/63 (76.2\%), dizziness 23/24 (95.8\%); peripheral edema, 3/4 (75.0\%)]. Overall, 15 cases of somnolence (15/ $63,23.8 \%)$ and one case of peripheral edema (1/ $4,25.0 \%$ ) were moderate and there was one case of severe dizziness $(1 / 24,4.2 \%)$.

The results of the subgroup analysis except for peripheral edema are shown in Table S2 in the electronic supplementary material. Although no test was performed for statistical significance and the number of patients was

Table 3 Incidence of somnolence, dizziness, and peripheral edema (primary endpoint; safety analysis set)

\begin{tabular}{ll}
\hline & $\boldsymbol{N}=\mathbf{1 5 2}$ \\
\hline Somnolence & $63(41.4)$ \\
Mild & $48(31.6)$ \\
Moderate & $15(9.9)$ \\
Severe & $0(0.0)$ \\
Dizziness & $24(15.8)$ \\
Mild & $23(15.1)$ \\
Moderate & $0(0.0)$ \\
Severe & $1(0.7)$ \\
Peripheral edema & $4(2.6)$ \\
Mild & $3(2.0)$ \\
Moderate & $1(0.7)$ \\
Severe & $0(0.0)$ \\
\hline
\end{tabular}

Data are shown as $n(\%)$ 
biased, the incidences of somnolence were higher for patients with presence of somnolence at enrollment, for patients with concomitant use of tramadol and duloxetine, for patients without gradual pregabalin dose tapering, for patients who did not complete the treatment protocol (regardless of renal function), and for women. No such tendency was observed for dizziness.

The cumulative incidences of somnolence, dizziness, and peripheral edema are shown in Fig. 3. The cumulative incidence of somnolence from the time of enrollment to the end of the study showed that approximately half of the somnolence events occurred by day 4 , followed by another rise after the dose increase at day 8 , when the first dose increase of mirogabalin occurred, and approximately half of the dizziness events occurred from days 1 to 15 , when the second dose increase of mirogabalin occurred. The mean \pm SD maximum daily doses of mirogabalin in patients overall were $9.09 \pm 3.32 \mathrm{mg}, \quad 14.50 \pm 6.18 \mathrm{mg}, \quad$ and $22.00 \pm 8.12 \mathrm{mg}$, for days $1-7$, days $8-14$, and days $15-29$, respectively.

AEs and adverse drug reactions occurring in $\geq 2$ patients are shown in Table 4 . Overall, 90 patients (59.2\%) experienced at least one $\mathrm{AE}$ and 83 patients (54.6\%) experienced at least one adverse drug reaction. Twenty-one patients (13.8\%) discontinued treatment due to an $\mathrm{AE}$, of which $13(8.6 \%)$ and $8(5.3 \%)$ patients discontinued during and after the study period, respectively. AEs leading to treatment discontinuation occurring in two or more patients were somnolence [13 patients $(8.6 \%)$ ], dizziness [5 patients $(3.3 \%)]$, peripheral edema [2 patients $(1.3 \%)]$, and constipation [2 patients (1.3\%)]. All AEs leading to treatment discontinuation were considered to be related to mirogabalin. Two serious AEs not leading to discontinuation (lymphoma and cerebrovascular accident) were reported and a causal relationship between mirogabalin and the serious AEs was ruled out. No deaths were reported during the study period.

\section{Protocol Treatment Completion Status}

Regarding protocol treatment completion status by renal function, in patients with $\mathrm{CrCL} \geq 60 \mathrm{ml} / \mathrm{min}$, the status was complete in 76 patients $(50.0 \%)$ and incomplete in 38 patients $(25.0 \%)$. In those with CrCL 30 to $<60 \mathrm{ml} / \mathrm{min}$, the status was complete in 26

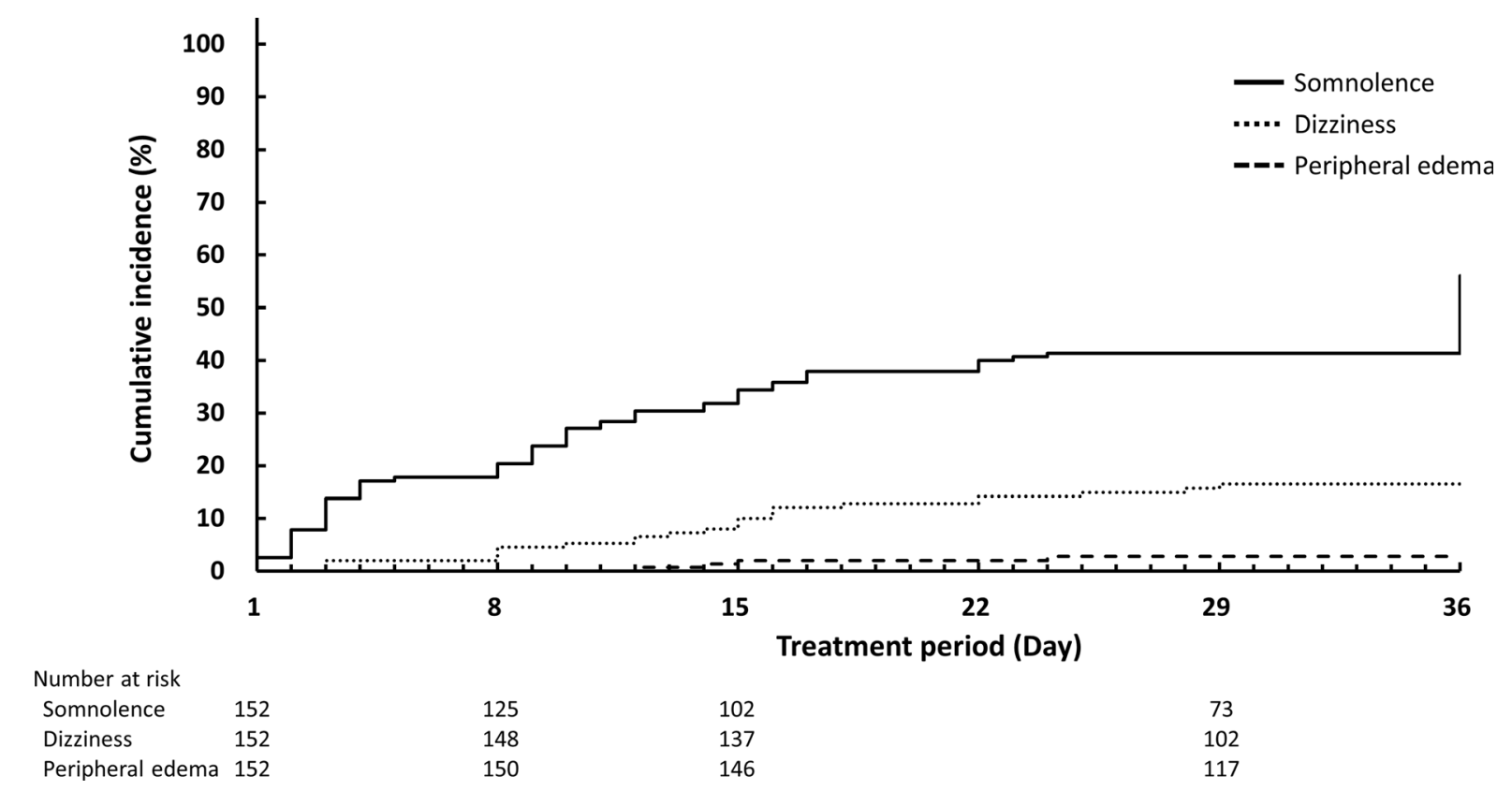

Fig. 3 Cumulative incidence of somnolence, dizziness, and peripheral edema (safety analysis set; $N=152$ ) 
Table 4 Adverse events and adverse drug reactions occurring in $\geq 2$ patients (safety analysis set)

\begin{tabular}{ll}
\hline & $N=\mathbf{1 5 2}$ \\
\hline Overall adverse events & $90(59.2)$ \\
Somnolence & $63(41.4)$ \\
Dizziness & $24(15.8)$ \\
Weight increased & $5(3.3)$ \\
Constipation & $4(2.6)$ \\
Peripheral edema & $4(2.6)$ \\
Nasopharyngitis & $3(2.0)$ \\
Headache & $3(2.0)$ \\
Fall & $3(2.0)$ \\
Decreased appetite & $2(1.3)$ \\
Loss of consciousness & $2(1.3)$ \\
Thirst & $2(1.3)$ \\
Overall adverse drug reactions & $83(54.6)$ \\
Somnolence & $63(41.4)$ \\
Dizziness & $23(15.1)$ \\
Weight increased & $5(3.3)$ \\
Constipation & $4(2.6)$ \\
Peripheral edema & $4(2.6)$ \\
Fall & $3(2.0)$ \\
Decreased appetite & $2(1.3)$ \\
Headache & $2(1.3)$ \\
Loss of consciousness & $2(1.3)$ \\
Thirst & $2(1.3)$ \\
\hline Dat & \\
\hline &
\end{tabular}

Data are shown as $n$ (\%)

Coded using the Japanese Medical Dictionary for Regulatory Activities (MedDRA/J), version 23.0

patients (17.1\%) and incomplete in 12 patients (7.9\%).

\section{Efficacy}

In the efficacy analysis set, the mean VAS score decreased from day $1(67.4 \pm 16.9 \mathrm{~mm})$ to day

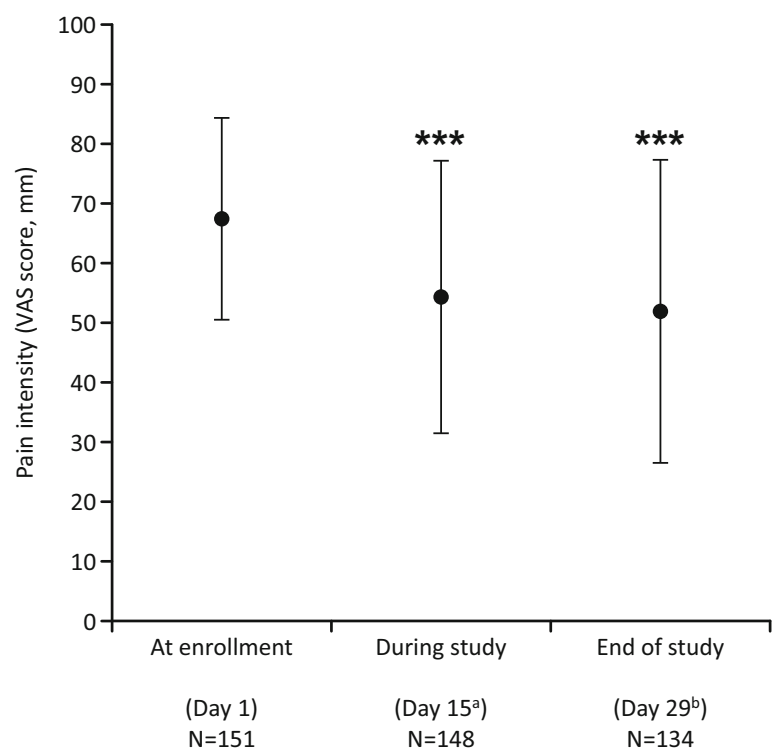

Fig. 4 Pain intensity (VAS score) from study enrollment to end of study (efficacy analysis set; $N=151$ ). Data are mean \pm standard deviation. ${ }^{a} \pm 7$ days; i.e., dose-adjustment period could begin anywhere between days 9 and 22 . ${ }^{b} \pm 7$ days; i.e., end of study occurred anywhere between days 22 and $36 .{ }^{* * *} p<0.001$ vs. baseline using a paired $t$ test. $V A S$ visual analog scale

$15 \quad(54.3 \pm 22.8 \mathrm{~mm})$ and day 29 $(51.9 \pm 25.4 \mathrm{~mm})$. Mean changes from day 1 to day 15 and day 29 were $-13.2 \pm 19.4 \mathrm{~mm}$ and $-15.7 \pm 24.1 \mathrm{~mm}$, respectively (both $p<0.0001$, Fig. 4 and Table 5). When analyzed by protocol treatment completion status (complete or incomplete), a significant decrease in VAS score was observed regardless of renal function except for the subgroup of patients with reduced renal function (CrCL 30 to $<60 \mathrm{ml} / \mathrm{min}$ ) who did not complete the study protocol (Table 5).

Significant reductions in VAS scores were observed at days 15 and 29, respectively, in patients with postherpetic neuralgia (both $p<0.0001)$, lumbar spinal stenosis $(p=0.0082$ and $p=0.0164$ ), and other peripheral neuropathic pain (both $p<0.0001$ ). Although a significant decrease in VAS score at day 15 in patients with lumbar disc herniation was observed $(p=0.0055)$, this change was not maintained at day $29(p=0.0983)$. 
Table 5 Changes in VAS scores according to VAS score at baseline (enrollment), treatment completion status, and renal function (efficacy analysis set)

\begin{tabular}{|c|c|c|c|c|}
\hline & & $\begin{array}{l}\text { Enrollment } \\
\text { (visit 1) }\end{array}$ & $\begin{array}{l}\text { During study } \\
\text { (visit 2) }\end{array}$ & $\begin{array}{l}\text { End of study } \\
\text { (visit 3) }\end{array}$ \\
\hline \multirow[t]{7}{*}{ Total } & $N$ & 151 & 148 & 134 \\
\hline & Mean \pm SD & $67.4 \pm 16.9$ & $54.3 \pm 22.8$ & $51.9 \pm 25.4$ \\
\hline & Range & $40-100$ & $7-100$ & $0-100$ \\
\hline & Median & $68.0(52.0,79.0)$ & $56.0(35.5,71.0)$ & $52.0(33.0,71.0)$ \\
\hline & (Q1, Q3) & & & \\
\hline & $\begin{array}{l}\text { Mean } \pm \text { SD change from } \\
\text { baseline }\end{array}$ & - & $-13.2 \pm 19.4$ & $-15.7 \pm 24.1$ \\
\hline & $p$ value & - & $<0.0001$ & $<0.0001$ \\
\hline \multicolumn{5}{|l|}{ Primary target disease } \\
\hline \multirow{7}{*}{$\begin{array}{l}\text { Diabetic peripheral neuropathic } \\
\text { pain }\end{array}$} & $n$ & 1 & 1 & 1 \\
\hline & Mean $\pm S D$ & 67.0 & 60.0 & 35.0 \\
\hline & Range & - & - & - \\
\hline & Median & - & - & - \\
\hline & (Q1, Q3) & & & \\
\hline & $\begin{array}{l}\text { Mean } \pm \text { SD change from } \\
\text { baseline }\end{array}$ & - & - & - \\
\hline & $p$ value & - & - & - \\
\hline \multirow[t]{7}{*}{ Postherpetic neuralgia } & $n$ & 43 & 42 & 38 \\
\hline & Mean $\pm S D$ & $63.5 \pm 16.0$ & $49.3 \pm 22.4$ & $45.6 \pm 25.1$ \\
\hline & Range & $42-99$ & $7-93$ & $5-86$ \\
\hline & Median & $60.0(48.0,77.0)$ & $50.0(31.0,68.0)$ & $45.5(28.0,67.0)$ \\
\hline & (Q1, Q3) & & & \\
\hline & $\begin{array}{l}\text { Mean } \pm \text { SD change from } \\
\text { baseline }\end{array}$ & - & $-13.9 \pm 15.9$ & $-16.9 \pm 18.4$ \\
\hline & $p$ value & - & $<0.0001$ & $<0.0001$ \\
\hline \multirow[t]{5}{*}{ Lumbar spinal stenosis } & $n$ & 41 & 40 & 32 \\
\hline & Mean $\pm S D$ & $64.5 \pm 16.7$ & $52.9 \pm 22.5$ & $51.4 \pm 25.4$ \\
\hline & Range & $40-100$ & $9-100$ & $11-100$ \\
\hline & Median & $65.0(51.0,77.0)$ & $53.5(35.0,67.5)$ & $56.5(30.5,71.5)$ \\
\hline & (Q1, Q3) & & & \\
\hline
\end{tabular}


Table 5 continued

\begin{tabular}{|c|c|c|c|c|}
\hline & & $\begin{array}{l}\text { Enrollment } \\
\text { (visit 1) }\end{array}$ & $\begin{array}{l}\text { During study } \\
\text { (visit 2) }\end{array}$ & $\begin{array}{l}\text { End of study } \\
\text { (visit 3) }\end{array}$ \\
\hline & $\begin{array}{l}\text { Mean } \pm \text { SD change from } \\
\text { baseline }\end{array}$ & - & $-11.6 \pm 26.3$ & $-13.2 \pm 29.4$ \\
\hline & $p$ value & - & 0.0082 & 0.0164 \\
\hline \multirow[t]{7}{*}{ Lumbar disc herniation } & $n$ & 11 & 11 & 9 \\
\hline & Mean $\pm S D$ & $70.4 \pm 19.3$ & $54.8 \pm 23.2$ & $59.3 \pm 27.2$ \\
\hline & Range & $42-98$ & $31-100$ & $30-99$ \\
\hline & Median & $69.0(51.0,91.0)$ & $48.0(33.0,75.0)$ & $50.0(34.0,88.0)$ \\
\hline & (Q1, Q3) & & & \\
\hline & $\begin{array}{l}\text { Mean } \pm \text { SD change from } \\
\text { baseline }\end{array}$ & - & $-15.5 \pm 14.6$ & $-11.9 \pm 19.1$ \\
\hline & $p$ value & - & 0.0055 & 0.0983 \\
\hline \multirow{7}{*}{$\begin{array}{l}\text { Other peripheral neuropathic } \\
\text { pain }\end{array}$} & $n$ & 55 & 54 & 54 \\
\hline & Mean \pm SD & $72.1 \pm 16.5$ & $58.9 \pm 23.0$ & $55.8 \pm 25.1$ \\
\hline & Range & $40-100$ & $12-100$ & $0-99$ \\
\hline & Median & $74.0(61.0,84.0)$ & $62.0(38.0,77.0)$ & $59.0(36.0,73.0)$ \\
\hline & (Q1, Q3) & & & \\
\hline & $\begin{array}{l}\text { Mean } \pm \text { SD change from } \\
\text { baseline }\end{array}$ & - & $-13.5 \pm 17.2$ & $-16.6 \pm 25.3$ \\
\hline & $p$ value & - & $<0.0001$ & $<0.0001$ \\
\hline \multicolumn{5}{|l|}{ Protocol completion status } \\
\hline \multirow{7}{*}{$\begin{array}{l}\text { Complete }(\mathrm{CrCL}: \geq 60 \mathrm{ml} / \\
\min )\end{array}$} & $n$ & 76 & 76 & 76 \\
\hline & Mean $\pm S D$ & $67.9 \pm 17.8$ & $52.0 \pm 24.0$ & $49.7 \pm 26.4$ \\
\hline & Range & $40-100$ & $7-100$ & $0-100$ \\
\hline & Median & 68.0 & 51.5 & 50.0 \\
\hline & (Q1, Q3) & $(51.0,82.5)$ & $(33.0,68.0)$ & $(29.0,68.0)$ \\
\hline & $\begin{array}{l}\text { Mean } \pm \text { SD change from } \\
\text { baseline }\end{array}$ & - & $-15.8 \pm 18.3$ & $-18.2 \pm 25.1$ \\
\hline & $p$ value & - & $<0.0001$ & $<0.0001$ \\
\hline
\end{tabular}


Table 5 continued

\begin{tabular}{|c|c|c|c|c|}
\hline & & $\begin{array}{l}\text { Enrollment } \\
\text { (visit 1) }\end{array}$ & $\begin{array}{l}\text { During study } \\
\text { (visit 2) }\end{array}$ & $\begin{array}{l}\text { End of study } \\
\text { (visit 3) }\end{array}$ \\
\hline \multirow{7}{*}{$\begin{array}{l}\text { Incomplete }(\mathrm{CrCL} \geq 60 \mathrm{ml} / \\
\min )\end{array}$} & $n$ & 37 & 35 & 26 \\
\hline & Mean $\pm S D$ & $68.6 \pm 16.2$ & $57.5 \pm 22.7$ & $56.2 \pm 22.9$ \\
\hline & Range & $42-100$ & $9-100$ & $11-96$ \\
\hline & Median & 69.0 & 57.0 & 55.0 \\
\hline & (Q1, Q3) & $(56.0,77.0)$ & $(42.0,72.0)$ & $(37.0,73.0)$ \\
\hline & $\begin{array}{l}\text { Mean } \pm \text { SD change from } \\
\text { baseline }\end{array}$ & - & $-11.3 \pm 20.6$ & $-13.2 \pm 20.3$ \\
\hline & $p$ value & - & 0.0027 & 0.0028 \\
\hline \multirow{7}{*}{$\begin{array}{l}\text { Complete (CrCL: } 30 \text { to } \\
<60 \mathrm{ml} / \mathrm{min})\end{array}$} & $\mathrm{n}$ & 26 & 26 & 26 \\
\hline & Mean $\pm S D$ & $66.9 \pm 17.1$ & $55.2 \pm 21.7$ & $50.5 \pm 26.1$ \\
\hline & Range & $42-98$ & $14-100$ & $6-86$ \\
\hline & Median & 65.0 & 57.5 & 59.0 \\
\hline & (Q1, Q3) & $(50.0,82.0)$ & $(40.0,72.0)$ & $(28.0,71.0)$ \\
\hline & $\begin{array}{l}\text { Mean } \pm \text { SD change from } \\
\text { baseline }\end{array}$ & - & $-11.7 \pm 22.1$ & $-16.4 \pm 23.8$ \\
\hline & $p$ value & - & 0.0122 & 0.0017 \\
\hline \multirow{7}{*}{$\begin{array}{l}\text { Incomplete (CrCL: } 30 \text { to } \\
<60 \mathrm{ml} / \mathrm{min})\end{array}$} & $n$ & 12 & 11 & 6 \\
\hline & Mean $\pm S D$ & $62.3 \pm 12.8$ & $57.0 \pm 16.9$ & $68.3 \pm 14.7$ \\
\hline & Range & $46-80$ & $29-80$ & $44-83$ \\
\hline & Median & 65.5 & 60.0 & 70.0 \\
\hline & (Q1, Q3) & $(49.0,74.5)$ & $(41.0,70.0)$ & $(62.0,81.0)$ \\
\hline & $\begin{array}{l}\text { Mean } \pm \text { SD change from } \\
\text { baseline }\end{array}$ & - & $-5.1 \pm 15.0$ & $8.2 \pm 15.0$ \\
\hline & $p$ value & - & 0.2859 & 0.2409 \\
\hline
\end{tabular}

$C r C L$ creatinine clearance, $Q$ quartile, $S D$ standard deviation, $V A S$ visual analog scale

\section{DISCUSSION}

This is the first multi-center, prospective study to assess the safety and efficacy of switching from pregabalin to mirogabalin without washout in patients with peripheral neuropathic pain. The incidences of somnolence, dizziness, and peripheral edema during the 4-week observation period were 41.4, 15.8, and $2.6 \%$, of which $>70 \%$ cases were mild in 
severity. The incidence of these AEs increased as the mirogabalin dose increased during the study. Discontinuation due to AEs was observed in ten patients. Overall, we found no new major safety concerns regarding the switch from pregabalin to mirogabalin, which involved tapering of the pregabalin dose (in accordance with the package insert), ahead of a step-wise titration of the mirogabalin dose under clinical practice conditions. Most patients (> 70\%) were able to continue study treatment, escalating to an effective dose of mirogabalin (10 or $15 \mathrm{mg}$ BID for patients with normal renal function and mild renal impairment, or 5 or $7.5 \mathrm{mg}$ BID for patients with moderately impaired renal function). Pain appeared to be well managed during the switch from pregabalin to mirogabalin, with significant reductions in mean VAS pain scores observed from baseline to the end of the study. Unlike other studies [7, 8], there was no washout period, so patients underwent a straightforward transition from pregabalin to mirogabalin with no break in treatment.

Although more detailed examination is required, our results suggest that it may be necessary to monitor certain patients more closely when switching from pregabalin to mirogabalin in real-world clinical practice, to reduce the risk of AEs. Such events were more likely to occur at the start of the switch to mirogabalin or at the beginning of the mirogabalin dose increase. Thus, more careful monitoring is needed immediately after switching to mirogabalin. In particular, careful monitoring may be even more important in women, in patients with pre-existing somnolence or dizziness, in patients switching from low-dose maintenance pregabalin, or when patients are prescribed certain concomitant medications such as tramadol or duloxetine.

Mirogabalin is predominantly renally excreted and undergoes minimal in vivo metabolism; thus, the drug-drug interaction risk is considered low. In healthy individuals, concomitant tramadol had little effect on the pharmacokinetics of mirogabalin, nor did it affect the incidences of somnolence or dizziness [17]. In clinical situations, the incidences of somnolence and dizziness have been shown to be higher when pregabalin was used in combination with opioids such as tramadol [18]. Further studies are needed regarding the combined use of these drugs in clinical practice.

In two double-blind, placebo-controlled phase 3 studies, somnolence occurred in $8.5-23.9 \%$ of patients across the $15-30 \mathrm{mg}$ /day mirogabalin dose range $[7,8]$. In our study, the incidence of somnolence (41.4\%) was higher. In contrast, the incidences of end-of-study dizziness $(15.8 \%)$ and peripheral edema $(2.6 \%)$ were similar to those of previous studies, which reported incidences of $4.9-15.5$ and $1.3-8.5 \%$, respectively. It is not fully understood why the rate of somnolence was higher in the present study; however, one possibility is that it may be due to the abovementioned concomitant use of tramadol with mirogabalin, or the high baseline rate of somnolence (16.4\%). Furthermore, in this study, patients were asked to report AEs at each visit, which may have led to the collection of more detailed event occurrence information, increasing the reported incidence.

Mirogabalin was effective at reducing VAS pain scores in patients with peripheral neuropathic pain, with significant reductions in VAS scores reported across all study visits in patients with both moderate ( 40 to $<60 \mathrm{~mm}$ ) and high ( $\geq 60 \mathrm{~mm}$ ) pain levels at study enrollment. The change in VAS score exceeded $10 \mathrm{~mm}$ both after 2 weeks and after 4 weeks. These changes are considered a minimal clinically important difference in accordance with the Initiative on Methods, Measurement, and Pain Assessment in Clinical Trials [19]. In our study, we identified improvements in VAS scores at day 15. Previous phase 3 studies of mirogabalin have shown improvements in VAS scores as early as 1 week after initiating treatment, although these studies did not involve switching pain medications $[7,8]$.

No significant decrease in VAS score was observed in patients with reduced renal function (CrCL 30 to $<60 \mathrm{ml} / \mathrm{min}$ ) who did not fully complete the treatment protocol. By the end of the study, the mean maximum daily dose of mirogabalin in these patients was $12.88 \mathrm{mg}$, which was lower than in the other subgroups. Therefore, an improvement in VAS score may not have been achieved because the 
effective dose of mirogabalin could not be administered.

With regard to disease type, a significant decrease in VAS score was observed in patients with postherpetic neuralgia, lumbar spinal stenosis, and other peripheral neuropathic pain. This suggests that mirogabalin may also be an effective therapeutic agent for peripheral neuropathic pain other than diabetic peripheral neuropathic pain and postherpetic neuralgia, effects that have been verified in previous phase three studies $[7,8]$. To our knowledge, our study is the first to suggest the effectiveness of mirogabalin for peripheral neuropathic pain other than diabetic peripheral neuropathic pain and postherpetic neuralgia. Pregabalin has not been reported to have an analgesic effect for neuropathic pain in lumbar spinal stenosis or herniated lumbar disc, but pain-relieving effects for neuropathic pain related to radiculopathy have been reported [20]. This supports the idea that mirogabalin, which similarly acts as an $\alpha_{2} \delta$ ligand, may be effective for treating neuropathic pain other than diabetic peripheral neuropathic pain and postherpetic neuralgia.

Our results are generally aligned with previous studies. In a retrospective study by Tetsunaga et al. switching from pregabalin to mirogabalin due to AEs or a lack of efficacy was shown to be effective, with significant reductions in numeric rating scale scores reported after 1 week of treatment with mirogabalin [13]. However, in contrast with our study, the incidences of somnolence $(26.7 \%)$, dizziness $(12.3 \%)$, and edema $(5.9 \%)$ were lower in the study by Tetsunaga et al. This difference may be due to the mean maximum dose of mirogabalin and the time from pregabalin discontinuation to mirogabalin switch. The mean maximum dose of mirogabalin was approximately three times higher (20.9 vs. $6.4-6.6 \mathrm{mg} /$ day) in our study. In addition, our study did not include a washout period between treatments, whereas the previous study included a washout period of at least 1 month. Moreover, the results from our study, with its high average maximum dose, suggest that the efficacy of mirogabalin may be increased as the dose increases, because VAS scores improved along with mirogabalin dose increases. In contrast, the previous study did not show dose-dependent efficacy of mirogabalin, possibly due to the lower average doses used.

The present study has some limitations. The absence of masking in this open-label, singlearm study may have introduced bias, and the lack of a placebo control means that we are unable to determine possible placebo effects of switching to a new medication. This study was restricted to 10 centers in Japan; thus, findings may not be applicable to other countries. Our study assessed the switch from pregabalin to mirogabalin in patients with peripheral neuropathic pain with VAS score $\geq 40 \mathrm{~mm}$. The findings may not be applicable to other patients, such as those with pain ratings $<40 \mathrm{~mm}$ with pregabalin. Finally, the short study period (4 weeks) does not provide any insights on the long-term safety and efficacy of switching from pregabalin to mirogabalin. Outcomes of AEs occurring after the end of the observation period could not be followed up in this study. Notably, weight gain is known to be a common $\mathrm{AE}$ with respect to mirogabalin $[7,8]$ but because this would be difficult to detect in a study of only 4 weeks, this AE was not included in the primary endpoint.

\section{CONCLUSIONS}

In patients with peripheral neuropathic pain, switching from pregabalin to mirogabalin (following pregabalin dose tapering and a step-wise titration of mirogabalin) was generally well tolerated and effective in pain management. In the clinical practice setting, most AEs were mild, and few patients discontinued the study. Most patients were able to undergo dose titration to an effective dose. Further studies are required to determine the long-term efficacy and tolerability of this switching protocol.

\section{ACKNOWLEDGEMENTS}

We thank the participants of the study. 
Funding. This study was funded by Daiichi Sankyo, Co., Ltd., which also provided funding for fees associated with medical writing support and the journal's Rapid Service Fee.

Medical Writing Assistance. We thank Emma Donadieu, MSc, and Mary Richardson, MSc, of Edanz Evidence Generation, for providing medical writing support, which was funded by Daiichi Sankyo Co., Ltd.

Authorship. All named authors meet the International Committee of Medical Journal Editors (ICMJE) criteria for authorship for this article, take responsibility for the integrity of the work as a whole, and have given their approval for this version to be published.

Authors' Contributions. Conceptualization: YKi, SY, JK, NH, HT, YKe, KS, MI, Methodology: YKi, SY, JK, NH, HT, YKe, KS, MS, MI, Investigation: YKi, SY, TS, JK, SC, NH, KY, YT, MI, Formal analysis: MS, Validation: MS, Writing Original Draft: YKi, Writing - Review \& Editing: All authors.

Prior Presentation. This research was presented at the 42nd Annual Meeting of the Japanese Association for the Study of Pain (4-5 December 2020, Tokyo, Japan).

Disclosures. Yoshiyuki Kimura has received lecture fees from Daiichi Sankyo Co., Ltd. Shigeki Yamaguchi has received lecture and manuscript writing fees from Daiichi Sankyo Co., Ltd. Takahiro Suzuki has received lecture fees from MSD K.K., Shionogi Co., Ltd., Eli Lilly Japan K.K., and Ayumi Pharmaceutical Corporation; and research funding from Shionogi Co., Ltd. Jitsu Kato has received lecture fees from Daiichi Sankyo Co., Ltd. Satoko Chiba has received lecture fees from Daiichi Sankyo Co., Ltd. Naomi Hirakawa has received lecture fees from Daiichi Sankyo Co., Ltd. Keisuke Yamaguchi has received lecture fees from Daiichi Sankyo Co., Ltd. Yutaka Tanabe has received lecture fees from Nippon Zoki Pharmaceutical Co., Ltd. Hiroshi Takatsuna is a full-time employee of the study sponsor, Daiichi Sankyo, Co., Ltd. Yusuke Kenyoshi is a full-time employee of the study sponsor, Daiichi Sankyo, Co., Ltd. Kazuhito Shiosakai is a full-time employee of the study sponsor, Daiichi Sankyo, Co., Ltd. Miyoshi Sakai is a full-time employee of EP-CRSU Co., Ltd. Masako Iseki has received lecture fees from Daiichi Sankyo Co., Ltd.

Compliance with Ethics Guidelines. The trial was conducted in accordance with the ethical principles, clinical research laws, and relevant notifications stipulated in the Declaration of Helsinki (as revised in 2013). The study received approval from the Clinical Research Review Committee, Itabashi Hospital, Nihon University School of Medicine (CRB No. CRB3180013), which notified all participating centers of the approval. This study was registered at the Japan Registry of Clinical Trials under the identifier jRCTs031190113. All patients provided written informed consent.

Data Availability. The datasets generated during and/or analyzed during the current study are not publicly available, but are available from the corresponding author on reasonable request.

Open Access. This article is licensed under a Creative Commons Attribution-NonCommercial 4.0 International License, which permits any non-commercial use, sharing, adaptation, distribution and reproduction in any medium or format, as long as you give appropriate credit to the original author(s) and the source, provide a link to the Creative Commons licence, and indicate if changes were made. The images or other third party material in this article are included in the article's Creative Commons licence, unless indicated otherwise in a credit line to the material. If material is not included in the article's Creative Commons licence and your intended use is not permitted by statutory regulation or exceeds the permitted use, you will need to obtain permission directly from the copyright holder. To view a copy of this licence, visit http://creativecommons.org/licenses/bync/4.0/. 


\section{REFERENCES}

1. Jensen TS, Baron R, Haanpää M, et al. A new definition of neuropathic pain. Pain. 2011;152(10):2204-5.

2. Galluzzi KE. Managing neuropathic pain. J Am Osteopath Assoc. 2007;107(10 Suppl 6):39-Es48.

3. O'Connor AB. Neuropathic pain: quality-of-life impact, costs and cost effectiveness of therapy. Pharmacoeconomics. 2009;27(2):95-112.

4. Finnerup NB, Attal N, Haroutounian S, et al. Pharmacotherapy for neuropathic pain in adults: a systematic review and meta-analysis. Lancet Neurol. 2015;14(2):162-73.

5. Freynhagen R, Baron R, Gockel U, Tölle TR. painDETECT: a new screening questionnaire to identify neuropathic components in patients with back pain. Curr Med Res Opin. 2006;22(10):1911-20.

6. Deeks ED. Mirogabalin: first global approval. Drugs. 2019;79(4):463-8.

7. Kato J, Matsui N, Kakehi Y, Murayama E, Ohwada S, Sugihara M. Mirogabalin for the management of postherpetic neuralgia: a randomized, doubleblind, placebo-controlled phase 3 study in Asian patients. Pain. 2019;160(5):1175-85.

8. Baba M, Matsui N, Kuroha M, Wasaki Y, Ohwada S. Mirogabalin for the treatment of diabetic peripheral neuropathic pain: a randomized, double-blind, placebo-controlled phase III study in Asian patients. J Diabetes Investig. 2019;10(5):1299-306.

9. Domon $\mathrm{Y}$, Arakawa $\mathrm{N}$, Inoue $\mathrm{T}$, et al. Binding characteristics and analgesic effects of mirogabalin, a novel ligand for the $\alpha(2) \delta$ subunit of voltage-gated calcium channels. J Pharmacol Exp Ther. 2018;365(3):573-82.

10. Baidya DK, Agarwal A, Khanna P, Arora MK. Pregabalin in acute and chronic pain. J Anaesthesiol Clin Pharmacol. 2011;27(3):307-14.

11. Pfizer. Lyrica (Pregabalin) Capsules $25 \mathrm{mg} 75 \mathrm{mg}$ $150 \mathrm{mg}$ Package Insert. 2020. Oct. Ver. 1. URL: http://labeling.pfizer.com/showlabeling.aspx?id= 561. Accessed 23 Mar 2021.
12. Shimada $Y$, Inage $K$, Orita $S$, et al. Effect of duloxetine on neuropathic pain in patients intolerant to continuous administration of pregabalin. Spine Surg Relat Res. 2017;1(1):40-3.

13. Tetsunaga $T$, Tetsunaga $T$, Nishida $K$, et al. Shortterm outcomes of mirogabalin in patients with peripheral neuropathic pain: a retrospective study. J Orthop Surg Res. 2020;15(1):191.

14. Haefeli M, Elfering A. Pain assessment. Eur Spine J. 2006;15(Suppl 1):S17-24.

15. Japan Society of Pain Clinicians. Guidelines for the Pharmacologic Management of Neuropathic Pain. Second Edition. 2019. http://minds4.jcqhc.or.jp/ minds/Pharmacologic-management-of-neuropathic -pain/Pharmacologic-management-of-neuropathicpain.pdf. Accessed 17 Feb 2021.

16. Treede RD, Jensen TS, Campbell JN, et al. Neuropathic pain: Redefinition and a grading system for clinical and research purposes. Neurology. 2008;70(18):1630-5.

17. Jansen M, Mendell J, Currie A, et al. Pharmacokinetics, pharmacodynamics, safety, and tolerability of mirogabalin when coadministered with lorazepam, zolpidem, tramadol, or ethanol: results from drug-drug interaction studies in healthy subjects. Clin Pharmacol Drug Dev. 2018;7(6):597-612.

18. Ohishi A, Chisaki Y, Hira D, Nagasawa K, Terada T. Opioid analgesics increase incidence of somnolence and dizziness as adverse effects of pregabalin: a retrospective study. J Pharm Health Care Sci. 2015;1:30.

19. Dworkin RH, Turk DC, Farrar JT, Haythornthwaite JA, Jensen MP, Katz NP, et al. Core outcome measures for chronic pain clinical trials: IMMPACT recommendations. Pain. 2005;113(1-2):9-19.

20. Saldaña MT, Navarro A, Pérez C, Masramón X, Rejas J. Patient-reported-outcomes in subjects with painful lumbar or cervical radiculopathy treated with pregabalin: evidence from medical practice in primary care settings. Rheumatol Int. 2010;30(8): 1005-15. 